\title{
Acerca de los esbozos para una teoría filogenética kantiana (según la Crítica de la Facultad de Juzgar $\$ 80$ y 81$)^{*}$
}

\author{
On the Sketches for a Kantian Phylogenetic Theory \\ (according to the Critique of the Power of Judgment $\S \S 80$ and 81)
}

Natalia Andrea Lerussi

\begin{abstract}
Resumen
En el siguiente trabajo expondremos la teoría filogenética que se deja rastrear en la segunda parte de la Crítica de la Facultad de Juzgar dedicada a una crítica de la facultad de juzgar teleológica. Mostraremos que Kant presenta allí -en forma de esbozos- una concepción tanto del origen de lo orgánico como del mecanismo específico de producción de variaciones de lo orgánico, es decir, de las especies y subespecies. El tratamiento de la cuestión filogenética revelará la forma polémica, rigurosa y, en parte, original con la que el filósofo enfrentó el fenómeno de la vida.
\end{abstract}

Palabras clave: Kant - teleología - filogénesis - vida

\begin{abstract}
In the following paper I expound the phylogenetic theory outlined in the second part of the Critique of the Power of Judgment devoted to a critique of the teleological judgment. I show that Kant presents there a conception not only of the origin of the organic material, but also of the specific mechanism of production of variations of the organic material, that is to say, the origin of species and subspecies. The treatment of the phylogenetic question will reveal the polemic, rigorous and partially original way in which the philosopher faced the phenomenon of life.
\end{abstract}

Keywords: Kant - teleology - phylogenesis - life

\footnotetext{
* Recibido: 14 de Junio de 2012. Aceptado: 29 de Septiembre de 2012.

$\dagger$ Universidad Nacional de General Sarmiento, Consejo Nacional de Investigaciones Científicas y Técnicas.

Para contactar a la autora, por favor, escriba a: nlerussi@hotmail.com

Metatheoria 3(1)(2012): 73-92. ISSN 1853-2322.

(C) Editorial de la Universidad Nacional de Tres de Febrero. Publicado en la República Argentina.
} 


\section{Introducción}

The dispute over Kant's legacy in philosophy and literature, after all, does not resolve around the possibility of a priori geometry or physics, but around the possibility of a philosophical comprehension of the phenomena of life.

Helmut Müller-Sievers

Poco extendidas en la obra publicada y poco exploradas por los intérpretes son las ideas de Kant acerca del fenómeno de la vida. Nos referimos, específicamente, no a la cuestión en torno al modo de comprensión o enjuiciamiento de los cuerpos organizados en un nivel epistemológico o hermenéutico, que tiene un puesto específico en la segunda parte de la Crítica de la Facultad de Juzgar (CFJ) y fue objeto de recepciones de diferente naturaleza desde su aparición hasta el presente, sino a la teoría protocientífica -que se presenta en la forma de esbozos- tanto del origen como de la variación de lo orgánico, esto es, la cuestión filogenética. Este asunto es abordado, muy puntualmente, en dos parágrafos de la CFJ, correspondientes a la "Metodología de la Crítica de la facultad de juzgar teleológica", secciones $\$ \S 80$ y 81. Allí encontramos ciertos argumentos centrales, ciertas discusiones polémicas y la perspectiva, en parte original, con la que Kant enfrentó el fenómeno de la vida. De la dilucidación de lo allí implicado nos ocuparemos en el presente trabajo.

A continuación analizaremos, en primer lugar (1) que, según Kant, la comprensión del origen y desarrollo de los seres organizados, en el nivel filogenético, debe suponer una matriz originaria organizada (ursprüngliche Organization) y, consecuentemente, implica asumir que los seres organizados no solo por su forma y configuración actual sino también por su origen deben ser comprendidos según el principio de la finalidad. Esto significa, según veremos (1.1), que Kant se opone a la generación equivoca según la cual es posible explicar el origen de los seres organizados a partir de un material bruto sujeto a meras leyes mecánicas. En este primer nivel de análisis, mostraremos que una lectura completa de la fuente en la que se apoyan las interpretaciones que abogan por la reducción de la finalidad al mecanismo según Kant no son correctas y, por lo tanto, que se debe aceptar de manera exclusiva un tipo de producción o generación univoca de acuerdo con la cual el origen de un ser orgánico debe ponerse en un material que es, él mismo, orgánico u organizado. Ahora bien, la generación univoca puede dividirse, según analizaremos en el punto (1.2), en dos tipos: generación univoca homónima, un tipo de generación de la cual la experiencia da pruebas diariamente en la reproducción continua de las especies, y la generación univoca heterónima, en este segundo caso, se trata de la hipótesis aventurada pero no absurda según la cual la forma y/o configuración de los seres organizados es diferente a la forma y/o configuración de sus progenitores. El procedimiento por el cual la matriz orgánica originaria ha podido devenir en formas y configuraciones específicamente diferentes desde un mismo material orgánico será clarificado por nosotros en el punto (2). 
Según indicaremos en (2.1) las formas y configuraciones en las que varía la organización originaria, según Kant, deben remitirse a una concepción según la cual habría mecanismos específicos de producción de variaciones de lo orgánico en dos niveles, esto es, las especies y las subespecies. Así, en tensión con las lecturas que acentúan que el concepto de especie defendido por Kant atenta contra una concepción evolucionista o transformista de lo orgánico, mostraremos en esta sección cómo Kant delinea una teoría que da cuenta del surgimiento de nuevas especies en un momento anterior de la historia de la vida orgánica. Según el filósofo, la matriz originaria habría dado a luz, en un primer momento de actividad, diferentes formas ejemplares que, tarde o temprano, se habrían osificado en ciertas especies fijas; a partir de entonces, se detendría la producción de nuevas especies en la historia de la naturaleza orgánica. En el punto (2.2), finalmente, estudiaremos las diferentes escuelas que el filósofo pone en consideración a fin de comprender el origen y desarrollo de las subespecies. Específicamente, nos detendremos en la sección (2.2.1) a investigar la escuela que Kant suscribe, la teoría de la epigénesis, en la versión defendida por su contemporáneo Blumenbach (en el punto 2.2.1.1). Según la hipótesis central que presentaremos, finalmente en (2.2.1.2), Kant construye un modelo de epigénesis, en parte original, en el que confluyen los elementos incluidos por el científico natural de Göttingen en la comprensión de la producción de las variaciones de lo orgánico y un tercer elemento, extraño al modelo de epigénesis defendido por aquel, que es el de las disposiciones genéricas cuyo papel fundamental se deja clarificar a través de los textos de Kant sobre las diferentes razas humanas. ${ }^{1}$ Mediante la confluencia de estos elementos logra Kant comprender el modo como se producen las subespecies en el interior de las especies y concluye su aporte en lo que a la filogénesis concierne.

\section{Sobre la hipótesis de una organización originaria (ursprüngliche Organization)}

Según Kant, aunque la autorización para explicar todos los productos naturales según el principio mecánico es ilimitada, la capacidad para lograr dicha explicación

\footnotetext{
${ }^{1}$ El primer texto llevaba por título "Sobre las diferentes razas humanas" (1775/1777) y allí Kant anunciaba sus lecciones de geografía física. Como señala Zammito (2006, p. 37), se trata de un texto polémico que se encauzaba contra ciertas corrientes científico-filosóficas presuntamente poco serias y moralmente peligrosas que negaban la unidad de la especie humana. En el centro de la perspectiva kantiana sobre el origen de las razas se encuentra la defensa de aquella unidad a través de la tesis según la cual el ejemplar originario de la especie habría estado predispuesto a sufrir variaciones convenientes al clima y el lugar y devenido, consecuentemente, diferentes tipos humanos fisonómicamente distinguibles. La cuestión de las razas fue discutida por Kant en la década posterior en dos ocasiones más: en "Determinación del concepto de una raza humana" ("Determinación del concepto"), de 1785, y en "Sobre el uso de principios teleológicos en la filosofía" ("Sobre el uso"), de 1788, con cierta vehemencia retórica y es presumible que, según estima Zammito (2003, pp. 78-79), ese fuera el motivo que condujera a Kant a componer la segunda parte de la CFJ pues una teoría sobre la reproducción de la especie según diferentes razas requería una teoría sobre la (comprensión de la) vida orgánica, objeto de estudio principal de la "Crítica de la facultad de juzgar teleológica".
} 
en relación a los seres organizados es "muy restringida" y "claramente limitada" (AA V, p. 417). ${ }^{2}$ De esta manera, los seres organizados no solo pueden sino que deben ser comprendidos según el principio meramente subjetivo de la finalidad que define la labor de la facultad de juzgar teleológica -modo de comprensión alternativo al modo de explicación mecánico-. ${ }^{3}$

Ahora bien, Kant defiende en la CFJ sección $\$ 80$ la tesis según la cual la circunstancia de que el fundamento de comprensión de los seres organizados sea el principio de la finalidad tiene la consecuencia fundamental según la cual el origen de los seres organizados no puede ponerse en la materia bruta (esto es, en un material susceptible de ser explicado mecánicamente) sino, a la vez, en una organización originaria (ursprüngliche Organization). La naturaleza del fundamento de comprensión de ciertos seres implica asumir una cierta naturaleza de su origen. Si la forma y enlace de un ser organizado requiere de la introducción del concepto de finalidad, en el nivel de su comprensión actual, igualmente requiere de dicha introducción la comprensión del origen de aquel, en el nivel filogenético. De esta forma:

A fin de que el investigador de la naturaleza no trabaje a pura pérdida debe poner siempre en el fundamento del enjuiciamiento de las cosas, cuyo concepto en tanto fin natural está indudablemente bien fundado (los seres organizados), una cierta organización originaria que utilice aquel mecanismo propio para producir otras formas organizadas (organisierte Formen) o para desarrollar las suyas propias hacia nuevas configuraciones (zu neuen Gestalten). (AA V, p. 418)

La organización de la materia en los seres organizados no puede concebirse como el efecto azaroso del mecanismo de la materia bruta, sino como el producto de un ser que es resultado, a la vez, de una matriz originaria organizada. Un ser organi-

\footnotetext{
${ }^{2}$ Cabe aclarar que a continuación se consignarán los textos de Kant, exceptuando la Crítica de la razón pura (CRP), siguiendo la edición de las Obras completas de Kant de la Academia de Ciencias de Berlín indicada en la Bibliografía (véase Kant 1902ss). Estas se consignarán con las siglas AA, el número romano corresponde al tomo y el número arábico, a la página. La CRP se consignará, según el modo habitual: CRP, A (edición 1781) y/o B (edición 1787). Las traducciones al español tanto de los textos de la obra kantiana como de la literatura secundaria están bajo nuestra responsabilidad. Siempre que sea el caso, hemos indicado en la Bibliografía las traducciones al español que hemos consultado a la hora de realizar las nuestras.

${ }^{3}$ Entendemos, siguiendo a McLaughlin (1989, p. 138), que la explicación según el principio del mecanismo o mecánico es que, desde el punto de vista de una serie espacial, va de "las partes al todo". Lectura confirmada expresamente por Kant cuando afirma, al pasar, en CFJ que "nos representamos un modo de producción mecánico [...] si consideramos [...] un todo material, según su forma, como producto de sus partes y de sus fuerzas y facultades enlazada por sí misma” (AA V, p. 408). Dicho modo de explicación se opone, en el pensamiento del filósofo, a la comprensión o exposición según el nexo de la causalidad final (teleología) que implica una línea de dependencia causal inversa, esto es, desde la "idea del todo a las partes". En este sentido, McLaughlin (1989) afirma que se debe distinguir el principio del mecanismo tal como es expuesto por Kant en CFJ del principio de la causalidad de la naturaleza tal como es desarrollado en la Crítica de la razón pura (CRP). Como hemos señalado, la diferencia específica del mecanismo consiste en que "las partes determinan a todo; el todo no puede determinar a las partes". Por el contrario, en el concepto de causalidad de la naturaleza según CRP “está implicado una consecución en el tiempo y no un entrelazamiento en el espacio". Véase CRP B, p. 238/A, p. 193; B, p. 244/ A, p. 199; B, p. 301/A, p. 243; B, p. 246/ A, p. 201; B, p. 248/ A, p. 203. En la CFJ, la distinción entre causalidad eficiente (dentro de la cual se ubica, según el género, la causalidad mecánica) y causalidad final se encuentra en: AA V, p. 372. Hemos ofrecido una reconstrucción del argumento por el cual Kant justifica la necesidad de introducir el principio de la teleología en la investigación de la naturaleza en un artículo que nos permitimos referir al lector: Lerussi (2011).
} 
zado debe concebirse como producto de un ser que puede tener su misma u otra forma (Form), su misma u otra configuración (Gestalt) pero, en cualquier circunstancia, en tanto producto de un ser que posee una fuerza formadora ${ }^{4}$ singular de reproducir una forma o configuración, sea cual sea, es decir de organizarse.

\subsection{Contra la generación equívoca}

En este contexto, Kant introduce en una nota al pie de CFJ $\$ 80$ una distinción importante, ${ }^{5}$ cual es la diferencia entre generación equívoca (generatio aequivoca) y generación univoca (generatio univoca) de los seres organizados. Por la primera se entiende "la generación de un ser organizado a través del mecanismo de la materia bruta no organizada" (rohen unorganisierten Materie) y es sencillamente absurda (ungereimt). ${ }^{6}$ El filósofo, sin embargo, no ofrece aquí argumentos de por qué la generación equívoca no puede ser, ni siquiera, inteligida. Más aun, según defienden algunos intérpretes, unas líneas más debajo de las aquí referidas, Kant parece abiertamente defender la generación equivoca. ${ }^{7}$ El filósofo afirma:

\footnotetext{
${ }^{4}$ Se debe distinguir (véase AA V, p. 374) el concepto de fuerza motriz (bewegende Kraft) propio de las máquinas (como los relojes) del de fuerza formadora (bildende Kraft) propia de los seres orgánicos (como los árboles). El uso de la terminología, sin embargo, no está fijado en la CFJ. Pues, por ejemplo, en la sección §81 Kant distingue el concepto de impulso formativo (Bildungstrieb) que actuaría en la formación y reproducción de los seres organizados del concepto de una fuerza formativa (Bildungskraft) que es "meramente mecánica” (AA V, p. 424). Así, los términos de fuerza formativa y fuerza formadora tendrían un significado opuesto según la CFJ. En la sección $\$ 80$, además, Kant utiliza la noción de fuerza reproductora (Zeugungskraft) para referirse al tipo de fuerza que se les adscribe a los seres organizados (AA V, p. 419). Más allá del problema de la fijación de la terminología debemos tener en cuenta, entonces, que nos enfrentamos a dos conceptos de fuerza irreductibles, al menos en principio, el uno de tipo mecánico y el otro no susceptible de ser comprendido mecánicamente. Un comentario sobre la problemática conceptual aquí implicada se encuentra en Frigo (2009).
}

${ }^{5}$ Véase AA V, p. 420, nota de Kant.

${ }^{6}$ Véase, además, AA I, p. 227 y AA II, p. 125.

${ }^{7}$ McFarland (1970, p. 39) sostiene esta tesis tanto a partir de un pasaje de CRP (esto es: CRP B, p. 863/ A, p. 835) en relación al origen del sistema (de la razón) como a partir de la CFJ §78 (AA V, p. 411) y §80 (AA V, p. 420). Para este intérprete, según Kant, "la generatio aequivoca* [en latín en el original] es un absurdo excepto para el caso de los más bajos organismos”. La teoría de la generación equívoca es remitida por McFarland, a la vez, a la teoría de la generación espontánea "que se remonta por lo menos hasta Aristóteles y que continuó siendo sostenida hasta el siglo de Kant y posteriormente. Según esta teoría, los organismos simples podrían venir a la existencia directamente desde la materia inorgánica. [En correspondencia con esta teoría] Kant habla de gusanos siendo producidos como resultado de la descomposición de la materia [se refiere al pasaje remitido de CFJ §78]”. Los corchetes son añadido nuestro. Sin embargo, ninguno de los tres pasajes que este intérprete señala para sostener que Kant habría defendido la teoría en cuestión es suficiente para extraer dicha conclusión. En el pasaje de CRP Kant nos habla de la presunta generación equívoca del origen del sistema de la razón solo analógicamente; en CFJ §78 Kant menciona la tesis de la generación espontánea del gusano aunque no como tesis propia sino exclusivamente para mostrar que la admisión de esta es incompatible con una comprensión teleológica del origen de aquel. Esto es, en dicho pasaje Kant afirma la tesis de la incompatibilidad entre la regla del mecanismo y la de finalidad en la explicación de la génesis de los organismos y no, como supone McFarland, la regla del mecanismo en relación a los seres organizados. El pasaje de CFJ $\$ 80$ indicado por el intérprete, finalmente, expresamente niega por absurda la tesis de la generación equívoca. Ahora, en un pasaje que analizamos a continuación (esto es, en AA V, pp. 418-419) Kant parece sostener la generación equívoca. Como veremos, sin embargo, una justa comprensión contextual del pasaje muestra que ese no es el caso. Con todo, también defienden que Kant habría abogado por la generación equívoca: Stadler (1874, pp. 135-137), McLaughlin (2010). Según Lenoir (1980, p. 79), es Buffon quien paradigmáticamente en el siglo XVIII habría defendido la posibilidad de explicar mecánicamente las formas orgánicas. 
La analogía de las formas en tanto parecen ser producidas conforme a un arquetipo (Urbild) común a través de todas las diferencias, refuerza la suposición de un verdadero parentesco de estas en la generación desde una madre originaria común (Urmutter) a través de la cercanía gradual de una especie animal a otra, desde aquella en la que el principio de los fines parece estar mayormente acreditado, esto es, el hombre hasta el pólipo y de éste incluso hasta los musgos y líquenes y, finalmente, hasta los grados más bajos de la naturaleza que podemos advertir, hasta la materia bruta (rohen Materie). De ésta y de su fuerza parece derivarse (abzustammen scheint) toda la técnica de la naturaleza según leyes mecánicas. (AA V, pp. 418-419)

En la última frase de la cita Kant afirma expresamente que la entera técnica de la naturaleza, incluso los seres organizados, "parece derivarse" de la materia bruta no organizada y, por lo tanto, de un material explicable según leyes mecánicas. Así, Kant parece aquí comprometerse con la teoría que unas pocas líneas más arriba ha calificado de absurda. La forma y configuración del hombre sería el resultado, en última instancia, de la forma y configuración de estructuras muy simples (el pólipo, los musgos y líquenes) y estas, a la vez, resultado de la fuerza de la sola materia bruta. Se habilitaría, de este modo, la generación equívoca y, por lo tanto, también la posibilidad de reducir el principio de la finalidad al principio de la causalidad mecánica. ${ }^{8}$

Del contexto de esta cita problemática se deja extraer, sin embargo, el sentido exacto que Kant le adjudica. El arqueólogo de la naturaleza "puede dejar nacer" todas las criaturas de la materia bruta del seno de la tierra pero, tarde o temprano, "debe añadir a esta madre común [...] una organización dispuesta en conformidad a fin a todas estas criaturas" (AA V, p. 419). Así, cuando aquel arqueólogo insiste en la posibilidad de explicar o reducir los seres organizados a la fuerza de la materia bruta no habrá más que "demorado el fundamento de explicación un poco más allá (hat [...] weiter aufgeschoben)" puesto que, en realidad, "no puede arrogarse haber cumplimentado la generación de aquellos dos reinos [animal y vegetal] independientemente de la condición de las causas finales" (AA V, p. 420. Los corchetes son añadido nuestro). Kant señala que la reducción del reino orgánico al inorgánico en el contexto de la comprensión del origen de los primeros exige poner a la base del reino de lo inorgánico algún tipo de organización originaria; requiere,

\footnotetext{
${ }^{8}$ Más aun, de este pasaje algunos intérpretes han intentado extraer la tesis de un cierto "darwinismo" kantiano avant la lettre. Fundamentalmente véase: Stadler (1874, p. 141), Menzer (1911, p. 103), Roretz (1922, p. 137), Kleingeld (1995, p. 128), Gerhardt (2002, p. 251) y McLaughlin (2010). Los textos pioneros en la temática (que no hemos podido consultar) son: Schulze (1875) y Drews (1894). Arthur Lovejoy ([1911] 1959), sin embargo, ha mostrado en un artículo hoy clásico que Kant no puede ser considerado un precursor de Darwin "puesto que Darwin no fue el padre de la teoría de la evolución orgánica, que para la época de 1859 era ya una vieja aunque controvertida doctrina, sino el padre de la teoría de la selección natural en tanto explicación del origen de las especies. Esto es lo que Kant habría designado como una explicación puramente mecánica en contraste con una explicación teleológica. Y él era del todo hostil a la suposición según la cual cualquier fenómeno de la vida orgánica pueda ser explicado completamente de forma mecánica" (Lovejoy [1911] 1959, p. 175). Las itálicas son del autor. Como mostraremos a continuación, nuestra hipótesis de lectura sigue, en este punto, a la de A. Lovejoy. Esto es, entre otras cosas, porque el aparente mecanicismo kantiano en la explicación del origen de los seres orgánicos debe ser rechazado. De esta opinión también: Düsing (1968, p. 141), Löw (1986, pp. 183-188, véase p. 168), Zammito (1992, p. 189).
} 
consecuentemente, pensar lo inorgánico como orgánico. De este modo, una organización originaria es necesariamente presupuesta cuando nos enfrentamos al origen filogenético de los seres organizados. La generación equívoca no es correcta.

\subsection{Generación unívoca homónima y heterónima}

Además de la generación equívoca que rechaza, Kant expone dos tipos de generación unívoca según las cuales se produce "algo orgánico a partir de otra cosa orgánica”. Se trata de la generación unívoca homónima (generatio homonyma) o generación univoca heterónima (generatio heteronyma). La primera es la producción de un "producto que en la organización misma es del mismo tipo que la de su genitor" y la experiencia da a diario muestras de ella. Por otro lado, en la generación unívoca heterónima el ser organizado es producto de otro ser organizado cuya forma o configuración es diferente a la de él. En este caso, el producto "se diferencia específicamente" de su progenitor. Aunque no se hallan muestras en la experiencia de este último tipo de generación "no se contradice a priori en el juicio de la mera razón" que, por ejemplo, "ciertos animales acuáticos se transformen (ausbilden zu) poco a poco en animales palustres y estos, tras algunas generaciones, en animales terrestres” (AA V, p. 419). Gracias a este último tipo de generación, la afirmación según la cual solo es concebible para nosotros que los seres organizados sean producto de seres organizados no nos compromete con la tesis según la cual la naturaleza orgánica habría estado siempre definida según la forma y configuración tal como se nos presenta hoy a la mirada. Aunque sea una "atrevida aventura de la razón" (ein gewagtes Abenteuer der Vernunft) es perfectamente concebible que la forma y configuración específica de la naturaleza orgánica actual sea el resultado del desarrollo a través de siglos y milenios de una primera matriz orgánica. ${ }^{9}$ La causa de la diferencia en la forma o en la configuración de los descendientes de aquella matriz primera debe comprenderse mediante ciertos modelos conceptuales. Sobre ellos nos ocupamos a continuación.

\section{Generación unívoca heterónima}

\subsection{Sobre la producción de nuevas especies}

Arthur Lovejoy ([1911] 1959, p. 181) sostuvo que el concepto de especie defendido por $\mathrm{Kant}^{10}$ y por el cual pertenecen a una misma especie natural individuos que

\footnotetext{
${ }^{9}$ En sus recensiones al texto de Johann Gottfried von Herder Ideas de una filosofía de la historia de la humanidad, Kant juzga esta misma idea "tan monstruosa que la razón se retira de ella asustada" (AA VIII, p. 54); en "Sobre el uso", por otro lado, Kant señala que ella es "un juego entretenido" aunque infructuoso (AA VIII, p. 180); finalmente en la CFJ la misma idea gana el estatus de "hipótesis" de una razón atrevida (AA V, p. 420). Así, Kant parece haber ido progresivamente acercándose a la teoría de la generación heterónima (que en "Sobre el uso" Kant adjudica a Bonnet (AA VIII, p. 180)).

${ }^{10}$ Concepto que, según el mismo filósofo expresa en algunas ocasiones (AA II, p. 429, AA VIII, pp. 164-165), tomó de Buffon.
} 
pueden procrear descendencia fértil (AA II, p. 429 y AA VIII, pp. 164-165) es incompatible con una concepción que el intérprete llama "transformista" o "evolucionista" de las especies: "Si examinamos el modo como Kant define estas especies, descubrimos que su noción de ellas excluye expresamente la posibilidad de cualquier transformación de una en otra a través de los descendientes". ${ }^{11}$ Ahora bien, aunque este intérprete tenga razón en afirmar que el concepto de especie kantiano implica un anti-evolucionismo, es posible conjeturar, atendiendo a las fuentes, que Kant habría combinado dicho anti-evolucionismo de las especies, en el momento presente, con un transformismo en el momento originario del material orgánico, esto es, cuando no se habían establecido aún especies fijas. Según esta interpretación, Kant habría defendido que las especies no pueden transformarse en otras especies pero son el resultado del desarrollo o transformación de la organización originaria de la tierra antes de que hubiera especies.

Como se expresa en el párrafo del apartado $\$ 80$ de la CFJ, que copiamos en la sección (1) del presente trabajo, el arqueólogo de la naturaleza debe presuponer en el origen de los seres organizados una cierta organización originaria que se desarrolla y transforma, esto es, que "produ[ce] otras formas organizadas (organisierte Formen)" o "desarroll[a] las suyas propias hacia nuevas configuraciones (zu neuen Gestalten)" (se encuentra en: AA V, p. 418). Pero, ¿qué se está mentando aquí por aquellas "formas organizadas" y estas nuevas "configuraciones"? Proponemos entender que las "formas organizadas" en las que deviene la organización originaria de la naturaleza deben referirse a las formas genéricas o especies. Las "nuevas configuraciones" deben definir las formas específicas o subespecies. De este modo, la producción de una forma organizada o forma genérica del material orgánico originario sería equivalente a la institución de una nueva especie; en cambio, una modificación en la configuración de aquella consistiría en una desviación de una forma genérica y, por lo tanto, sería equivalente a la producción de una forma específica o subespecie.

Ahora, ¿qué factores intervendrían en la producción de las especies? Expresamente Kant especula, en CFJ §80, sobre los factores que actuarían en la formación de nuevas especies orgánicas desde una matriz originaria. El filósofo señala:

Él [el arqueólogo de la naturaleza] puede hacer nacer del seno materno de la tierra recién salida de su estado caótico (semejante a un gran animal), al principio, criaturas de forma menos ajustada a fin (Geschöpfer von minder zweckmäßiger Form), de éstas a su vez otras, que se darían forma (sich ausbildeten) de manera más adecuada a su lugar de producción (Zeugungsplatze) y a sus relaciones recíprocas (ihrem Verhältnisse unter einander); hasta que esta

${ }^{11}$ En esta línea, según Zammito (1992, p. 189, 2002, p. 306), la idea de evolución "asustaba" a Kant. Sin embargo, Lovejoy ([1911] 1959, p. 199), admite que la sección $\$ 80$ de la CFJ "es la fuente principal de la creencia de que Kant fue un evolucionista en biología" y que "es verdad que la posición de Kant se modificó significativamente en relación a los dos años previos". Más aun, "él ya no condena el transformismo sobre fundamentos a priori como una monstruosidad filosófica. Su verdad o falsedad se transforma en una cuestión que ha de ser resuelta a través de la evidencia empírica". 
matriz hubiera solidificado (sich erstarren), osificado y restringido sus partos a determinadas especies (Spezies) que no continúan degenerándose (ausarten) y la diversidad se mantendría como si ella [la tierra] hubiera suspendido al término de esta operación aquella fuerza formativa fecunda. (AA V, p. 419. Los corchetes son añadido nuestro)

En este pequeño fragmento Kant expone la manera como concibe el proceder de la organización originaria en la formación de las especies. Esta se daría formas progresivamente más ajustadas a fin en correspondencia con dos factores físicos externos, esto es, el lugar de producción (Zeugungsplatze) y las relaciones recíprocas entre los seres producidos (ihrem Verhältnisse unter einander). Ahora, llegado a un cierto punto de solidificación y osificación de sus producciones, la matriz originaria habría detenido su "degeneración" en ciertas formas genéricas o especies. Así, la producción de otras "formas organizadas", esto es, de nuevas especies, habría llegado a su fin y estas se reproducirían para siempre a partir de un ejemplar fijo. ${ }^{12}$

\subsection{Sobre la producción de nuevas subespecies}

Según Kant, hay en la historia natural dos escuelas principales para las cuales se debe suponer en el origen de los seres organizados una organización originaria y, con ella, el principio de la finalidad. Esto es, lo que él llama el "ocasionalismo" (Okkasionalism) y el "preestabilismo de las causas" (Prästabilism der Ursache) que se divide, a la vez, en "preformismo individual" o "teoría de la evolución" (Evolutionstheorie) y "preformismo genérico", "teoría de la involución" (Involutionstheorie) o "epigénesis" (Epigenesis). Puesto que todas estas tradiciones comparten la tesis, que Kant defiende, de la imposibilidad de explicar mecánicamente el origen de los seres organizados y, por lo tanto, todas exigen introducir el principio de la teleología en la comprensión de ellos, se debe clarificar tanto el sentido en el que estas divergen como el criterio para decidir cuál de ellas es la más adecuada para lo que se propone, es decir, para dar cuenta de la formación de variaciones o desviaciones específicas en el interior de las especies. Desde el punto de vista de Kant, el punto en el que aquellas desacuerdan es en el papel que le adjudican al principio mecánico en la comprensión de la producción de los seres organizados. Como veremos, el filósofo suscribirá el preformismo genérico, teoría de la involución o epigénesis porque, en contraste con el ocasionalismo y el preformismo individual, es la única escuela que le adjudica al mecanismo de la naturaleza un papel "inconfundible" (AA V, p. 424).

De acuerdo con la CFJ sección §81, el "ocasionalismo" supone que la "causa suprema del mundo" (oberste Weltursache) sin ninguna mediación "otorgaría [...] la

\footnotetext{
${ }^{12}$ Según Roth (2008, p. 279), la teoría de la generación univoca heterónima, en general, que Kant defendería en la CFJ, debe yacer en "su teoría del organismo". Conjetura del todo probable si notamos que la noción de ser organizado (AA V, p. 374, AA V, p. 376, AA VIII, pp. 179, 181) implica el de una fuerza formadora que comunica una forma tanto según un "ejemplar" pero también con "desviaciones convenientes que la autoconservación (Selbsterhaltung) exige de acuerdo con las circunstancias" (AA V, p. 374). En el corazón del concepto de lo orgánico, se encontraría el de especie (comunicación ejemplar) y el de subespecie (comunicación desviada de la fuerza formadora).
} 
formación orgánica (organisierte Bildung) a la materia mezclada en ocasión de cada apareamiento" (AA V, p. 422). La naturaleza no colaboraría, en absoluto, en la formación de la configuración específica de los seres organizados sino que esta sería el producto directo de una causa sobrenatural. Kant ironiza sobre esta tradición en tanto tiene que suponer que el coito "sería una mera formalidad" (AA V, p. 423) en la formación de un ser organizado. Así, el filósofo desecha rápidamente esta teoría en la medida en que ella suspende el uso de la razón en la comprensión de la naturaleza (organizada) y "nadie que tenga algo que ver con la filosofía admite dicho sistema" (AA V, p. 422). Por otro lado, Kant expone la teoría denominada "preestabilismo de las causas". Señala que, según esta escuela, la sabiduría suprema "habría colocado las disposiciones solo en los productos iniciales [...] mediante las cuales un ser organizado produce a su semejante” (AA V, p. 422). La intervención de lo sobrenatural según el "preestabilismo de las causas" sería menor, al menos en principio, que en el "ocasionalismo" pues es requerible solo en la producción de los primeros seres orgánicos. Las disposiciones en ellos colocadas harían el trabajo restante..$^{13}$ En este marco, Kant introduce una distinción relevante en el interior de dicho sistema puesto que el "preestabilismo de las causas" puede ser de dos tipos.

Según la "teoría de la evolución" o "preformación individual", la causa suprema del mundo habría producido cada embrión individual formado (gebildete Embryo) al comienzo del mundo y depositado en los primeros seres organizados. Así, se evitan las creaciones ocasionales en virtud del nacimiento de cada ser organizado. Sin embargo, las ventajas que puede arrogarse para sí el "preformismo individual" frente al "ocasionalismo" no son muchas y esto porque, aunque el teórico de la evolución pretenda asumir menos supuestos sobrenaturales que el ocasionalista, lo contrario es el caso, esto es:

como si no se ahorrase por medio de la creación ocasional una gran cantidad de dispositivos (Anstalten) sobrenaturales que serían requeribles para que el embrión formado en el comienzo del mundo no sucumba a las fuerzas destructivas de la naturaleza y se conserve ileso a través de un largo período hasta su desarrollo. (AA V, p. 423)

El "preformismo individual" no puede ser correcto no solo porque, como se indica en la cita, tiene que otorgarle un gran papel a lo sobrenatural para la protección de los primeros embriones contra las fuerzas destructivas de la naturaleza sino además porque, como señala el filósofo a continuación, este tipo de preformismo no puede dar cuenta del "nacimiento de monstruos" (Mißgeburen) y "bastardos" (Bastarden). ${ }^{14}$

\footnotetext{
${ }^{13}$ La definición de disposiciones y semillas se encuentra en el texto sobre las razas de los setenta en tanto "fundamentos de un cierto tipo de desarrollo" (AA II, p. 434) de un ser organizado.

${ }^{14}$ Véase AA V, p. 423. McLaughlin menciona cuatro factores fundamentales que socavaron las bases de la teoría de la evolución en la segunda mitad del siglo XVIII, esto es: "1) el desarrollo de teorías geológicas y cosmológicas; 2) el surgimiento de un criterio específico de especie; 3) la imposición de la filosofía atomista en la naturaleza y en la sociedad; 4) el surgimiento de un concepto general de un sistema orgánico” (McLaughlin
} 
Así, en la nomenclatura propuesta, solo resta ponderar la teoría del "preformismo genérico", "teoría de la involución” o "epigénesis". Kant no vacila en elogiar este último modelo y esto, no solo porque este parece tener "la gran ventaja" (AA $\mathrm{V}$, p. 424) de que la experiencia da pruebas de aquello que dice la teoría sino porque además "con el menor derroche posible de lo sobrenatural" (AA V, p. 424) deja a la naturaleza sola hacer el trabajo de reproducir la configuración o forma específica de los seres orgánicos. Desafortunadamente, sin embargo, no encontramos en la CFJ una exposición clara de aquello que Kant entiende por la teoría de la epigénesis. ${ }^{15}$ En la sección $\$ 81$ solo se encuentra un pasaje en el que Kant se refiere expresamente a ella en los siguientes términos:

Este último [sistema de la epigénesis] también puede llamarse sistema de la preformación genérica puesto que la capacidad productiva de los engendradores, esto es, la forma específica (die spezifische Form) estaba virtualmente preformada (virtualiter präformiert war) según las disposiciones internas conforme a fin que le fueron adjudicadas a su tronco. (AA V, p. 423. Los corchetes son añadido nuestro.)

Recordemos ahora que, según el "preestabilismo de las causas", una sabiduría suprema habría colocado las disposiciones de todos los seres orgánicos en los productos iniciales. Las disposiciones inscriptas en los seres organizados definen el arco de posibilidad de variación y, por lo tanto, excluyen que la transformación de un ser orgánico sea disparatada o arbitraria. Ahora, la variabilidad puede estar o bien genérica o bien individualmente prefigurada o preformada en las disposiciones originarias según el tipo de preformismo de que se trate. Según parece implicar aquí Kant, según el preformismo individual, la reproducción sería equivalente al alumbramiento de una semilla depositada en la simiente de la madre (semilla que, a la vez, habría sido heredada vía materna desde la primera forma originaria de cada especie $)^{16}$ que contiene la forma y configuración completa del futuro engen-

1989, pp. 16-20). Así, los descubrimientos geológicos señalaban que la tierra había sido habitada sucesivamente por especies distintas pero el preformismo (individual) supone que las semillas originarias fueron creadas simultáneamente en el primer minuto de la tierra. Por otro lado, aunque el criterio de especie impuesto por Buffon no estaba en contradicción, en principio, con el preformismo, este ponía al descubierto las artes bastardas cuya existencia no podría ser explicada mediante el preformismo. En tercer lugar, McLaughlin señala -aunque por razones que no hemos logrado descifrar- que el preformismo supone que la materia es infinitamente divisible, supuesto problemático a partir de la imposición del atomismo en física. El último y quizás más importante factor es el descubrimiento de la capacidad de reproducción del pólipo que condujo a un concepto de organismo como sistema con capacidad de autorreproducirse que no era explicable por el preformismo (individual). Con todo, Müller-Sievers (1997, pp. 5-6, 47) ha sostenido que el triunfo de la epigenénesis sobre el preformismo de finales del siglo XVIII no implicó, en realidad, ningún nuevo descubrimiento o experimento (Haller, de hecho, conocía ya la capacidad de reparación de los pólipos) ni ninguna evidencia microscópica particular, sino que se habría tratado de un "evento puramente textual" entendido como una "operación ideológica", una "revolución de paradigma" en el sentido kuhniano del término.

${ }^{15}$ Según señala Zammito (2003, p. 86), "hay un consenso notablemente pequeño sobre qué significa exactamente epigénesis en el discurso del siglo XVIII y no solo en Kant”.

${ }^{16}$ Que Kant esté aquí considerando un tipo de preformismo individual de tipo ovulista (preformación individual por vía materna) se sigue del señalamiento, al pasar, según el cual los representantes del preformismo individual "tuvieron que reconocerle al semen de la criatura masculina, a la que por lo demás no le habian reconocido sino la propiedad mecánica de servir de primer medio alimentario del embrión [...] una fuerza formativa” (AA V, p. 424). 
drado. La naturaleza del individuo estaría determinada en el embrión en la matriz de la primera madre desde el origen de las especies. Por eso, dice Kant que, según este tipo de preformismo, la naturaleza no tiene más que "desarrollar" las primeras semillas sin poder intervenir en ellas ni modificarlas. Por otro lado, según la epigénesis o preformismo genérico, la forma específica estaría preformada solo "virtualmente" en las disposiciones y semillas genéricas depositadas en los miembros de la estirpe originaria y sería el resultado del desarrollo diferencial de las disposiciones correspondientes. ¿Cuáles otros factores incidirían en la determinación de dicha diferenciación, esto es, en la producción de la forma específica de un ser orgánico según esta escuela? Ya que la conformación de la forma específica de un ser organizado no puede comprenderse apelando al concepto de disposiciones y semillas, dado que estas serían solo genéricas, la epigénesis requiere introducir otros elementos de comprensión. En la sección §81 no hay más aclaraciones al respecto. Pero la mención elogiosa del modelo de la epigénesis desarrollado por el científico natural Blumenbach nos ofrece una pista en la búsqueda de los conceptos requeridos.

\subsubsection{Epigénesis o preformismo genérico}

\subsubsection{El modelo de epigénesis desarrollado por Blumenbach}

Según nos señala Kant, Blumenbach rechazaría "con derecho" como "contrario a la razón" la posibilidad de explicar mecánicamente los seres organizados, "que la materia bruta se haya formado a sí misma originariamente según leyes mecánicas, que la vida haya surgido de la naturaleza de lo sin vida ([die] Natur des Leblosen)" (AA V, p. 424). ${ }^{17}$ La teoría de Blumenbach exige, consecuentemente, introducir el concepto de una fuerza irreductible al mecanismo natural aunque sin negarle por esto un "papel inconfundible" a él. Kant señala:

En vistas a esta teoría de la epigénesis, nadie ha prestado mayor servicio que el señor consejero de la corte Blumenbach, tanto en relación a la prueba como también en la fundamentación de los genuinos principios de su aplicación, en parte mediante la limitación de un uso desmedido de esta [...] Él deja al mecanismo natural $[\ldots]$ bajo este principio para nosotros inescrutable de una organización originaria, una participación indeterminable aunque al mismo tiempo inconfundible, por el cual se denomina a la facultad de la materia en un cuerpo organizado (a diferencia de la mera fuerza formativa (Bildungskraft) mecánica que, en general, habita en ella) impulso formativo (Bildungstrieb). (AA V, p. 424)

\footnotetext{
Las itálicas son añadido nuestro. Según nos enseña Löw (1986, p. 102), el ovulismo, que "supone que el óvulo es el organismo a desarrollarse y el espermatozoide debe dar solo el estímulo para su desarrollo", se opone al "animaculismo", esto es, la teoría que entiende que "el espermatozoide es, en realidad, el animal y el óvulo, solo el suelo alimentario de este". Ambas corrientes fueron las dos variantes principales de la teoría preformista en los siglos XVII y XVIII. Löw (1986) ubica entre los animaculistas, fundamentalmente, a pensadores de fines del siglo XVII y principios del siguiente, esto es, Leeuwenhoek, Hartsoeker, Leibniz, Boerhaave; entre los ovulistas, a pensadores fundamentalmente del XVIII, a saber Swammerdam, Malpighi, Vallisnieri, Bonnet, von Haller, Spallanzani.

${ }^{17}$ Véase Blumenbach (1791, pp. 79, 80).
} 
¿Qué está mentando aquí Kant por un "impulso formativo" y por una "participación inconfundible del mecanismo natural"? Puesto que en la CFJ no hay indicaciones al respecto, debemos dirigirnos al texto Sobre el impulso formativo de Blumenbach (1791), donde encontraremos una explicitación sobre lo mismo. La remisión al texto Sobre el impulso formativo parece aquí pertinente teniendo en cuenta que en 1789 , precisamente durante la redacción de la segunda parte de la CFJ, ${ }^{18}$ Blumenbach envió por correo una copia a Kant que el filósofo le agradeció un año después. ${ }^{19}$

El objetivo principal de Sobre el impulso formativo es, por un lado, refutar la "teoría de la evolución" y la necesidad del supuesto de los conceptos de disposiciones y semillas naturales en la comprensión de la producción de un cuerpo organizado y, por otro, proponer una teoría alternativa para dar cuenta de lo mismo, la teoría de la epigénesis. Así, según el científico, hay dos caminos principales para explicar la producción de un ser organizado, la teoría de la evolución, que él rechaza y la epigénesis, que cuenta con los mejores argumentos a su favor. Blumenbach (1791) escribe:

O bien se supone que el material productivo (Zeugunsstoffe) maduro, aunque bruto e informe de los padres, es desarrollado gradualmente hacia una nueva criatura cuando ha alcanzado su determinación según el tiempo y bajo las condiciones requeridas del lugar. Esto enseña la epigénesis. $\mathrm{O}$ bien se rechaza toda producción en el mundo y se cree, por el contrario, que para todos los hombres y animales y plantas que ya han vivido y que vivirán después, fueron creadas las semillas justo en el momento de la primera creación, de manera que ahora meramente se necesita de una generación tras otra para desarrollarlas. Por eso se llama a esta doctrina evolución. (Blumenbach 1791, p. 14)

A partir de la constatación de ciertos fenómenos naturales como la generación de nuevas especies ${ }^{20}$ y la constatación de bastardos, ${ }^{21}$ hermafroditas ${ }^{22} \mathrm{y}$

\footnotetext{
${ }^{18}$ Que Kant habría redactado la segunda parte de la CFJ recién durante el año 1789 es una estimación de Tonelli (1954, p. 445).

${ }^{19}$ Kant agradece a Blumenbach el envío efectuado "el año precedente" del manuscrito en la carta fechada el 5 de agosto de 1790. Véase AA XI, pp. 184-185. En esta carta Kant le dice a su corresponsal que "su trabajo lo ha instruido de muchas maneras, más aun, su reciente unificación de los dos principios, esto es, el físico-mecánico y el mero modo de explicación teleológico de la naturaleza organizada -que se tenían por incompatibles- tiene una relación cercana a las ideas que [l]e ocupan ahora, en primer lugar, pero que requieren de una tal confirmación fáctica" (AA XI, p. 185). Los corchetes son añadido nuestro. Nótese que la unificación entre los dos principios en cuestión, presuntamente realizada por la teoría de Blumenbach, parece definir el punto central del aplauso de Kant a ella. A continuación utilizaremos la edición de Sobre el impulso formativo de 1791 que es una versión exacta de la de 1789 (inconseguible). Una curiosidad, anotada por Löw (1986, pp. 104, 175), es que Kant no mencione jamás en toda su obra al epigenetista alemán más importante de la segunda mitad del siglo XVIII antes de Blumenbach, esto es, a Caspar F. Wolff.

${ }^{20}$ Blumenbach (1791, pp. 74-75), a diferencia de Kant, considera posible la producción de nuevas especies (Gattungen) en la actualidad. Así, comenta una experimentación realizada por el señor Kölreuter y descripta en su Transmutación completamente desarrollada de una especie vegetal natural en otra (Leipzig, 1761?) de la que habría resultado, a través de inseminación artificial, la reproducción repetida de una planta bastarda fértil y por la cual una especie (Gattung) de tabaco (Nicotiana rustica) mutaría en otro tipo (Nicotiana paniculata). Kant no menciona esta contra-prueba de la teoría de la evolución en la CFJ, probablemente porque consideraba que el experimento prueba menos de lo que pretende, esto es, no la producción de una nueva especie sino solo de una nueva variedad.

${ }^{21}$ Véase Blumenbach (1791, p. 74).

${ }^{22}$ Véase Blumenbach (1791, p. 98).
} 
monstruos, ${ }^{23}$ Blumenbach se propone demostrar que la teoría de la evolución ${ }^{24}$ no puede ser correcta. Esto significa que se debe mostrar "que no preexiste semilla preformada alguna" 25 en los cuerpos organizados. Por el contrario, se debe probar que un material productivo bruto e informe "cuando [...] ha alcanzado su determinación según el tiempo y bajo las condiciones requeridas del lugar" es desarrollado o formado a través de un "impulso vivo activo" (lebenslang thätiger Trieb). El modelo de epigénesis propuesto por Blumenbach se compone, consecuentemente, de dos elementos principales, esto es, el de los factores físicos externos de tiempo y lugar que direccionan las modificaciones y el concepto de una fuerza o impulso formativo que reproduce la modificación. Este impulso refiere a una fuerza de tipo física pero que se diferencia específicamente tanto de las "fuerzas físicas universales" como de "otras fuerzas vivas". ${ }^{26}$ Se trata de un impulso que:

pertenece a las fuerzas vivas (Lebenskräften) pero que se diferencia con claridad de otras fuerzas vivas de los cuerpos organizados (como la contractibilidad, la irritabilidad y la sensibilidad) como de las fuerzas físicas universales en general; que parece ser la primera fuerza más importante de toda producción (Zeugung), alimentación y reproducción (Reproduction) y que se puede denominar $[\ldots]$ con el nombre de impulso formativo (Bildungstrieb. nisus formatiuus). (Blumenbach 1791, p. 32)

La prueba principal tanto de la existencia del impulso formativo como de la inexistencia de semillas y disposiciones es la experimentación llevada a cabo por el autor con pólipos. Los experimentos mostraron que los pólipos, luego de ser mutilados, son capaces de reparar la pérdida mediante una reproducción de la parte ausente

${ }^{23}$ Véase Blumenbach (1791, pp. 111 y ss). El ejemplo de monstruosidad ofrecido son los cuerpos con dos cabezas.

${ }^{24}$ En lo relativo a esta teoría, Blumenbach (1791, p. 17) nos remite a los experimentos de Ludwig von Hammen, quien, en 1677, habría realizado un experimento con semen de gallo y descubierto, mediante el uso del microscopio, que en este habitan una innumerable cantidad de pequeños animales vivos que identificó como disposiciones y semillas.

${ }^{25}$ Véase Blumenbach (1791, p. 31).

${ }^{26}$ Blumenbach (1791, p. 32) subraya el carácter físico (es decir, no metafísico) de dicha fuerza cuando la equipara a la fuerza de atracción de Newton. Así, señala que se trataría de una fuerza cuyos "efectos constantes se reconocen en la experiencia pero cuya causa es -como la fuerza de atracción de Newton- una qualitatis occulta". Lenoir (1980, p. 83) ha señalado que una interpretación mecánico-natural del impulso formador no es adecuada para el caso de Blumenbach pero tampoco para el caso de Kant. Según aquel el "impulso formativo" "no es una fuerza mecánica ciega de expansión [...] no es una fuerza química de fermentación ni tampoco un alma sobreimpuesta a la materia. El Bildungstrieb* fue concebido como un agente teleológico que tendría sus antecedentes últimos en el reino de lo inorgánico pero que era una fuerza vital emergente”. Que el impulso formativo "tendría sus antecedentes últimos en el reino de lo inorgánico" no nos parece, sin embargo, una descripción correcta de la concepción kantiana según hemos visto arriba (en el apartado 1.1). De la opinión de que el impulso formador desarrollado por Kant no se reduce a las fuerzas mecánicas también es Jh. Zammito (2003, p. 82): "Yo sostengo que el lenguaje de Kant de semillas y disposiciones naturales y su aceptación de la idea de una Lebenskraft ${ }^{*}$ en tanto ejemplificada por el Bildungstrieb* de Blumenbach lo comprometió en una concepción de la ciencia de la vida que implica la realidad objetiva de fuerzas que no podrían ser reducidas a aquellas que él admitiría en el orden newtoniano o en la física". Esto significa, que si bien esta fuerza ha de concebirse en términos "físicos", no debemos reducirla a las fuerzas mecánicas de la naturaleza. La física se encontraría enriquecida, no empobrecida, por el descubrimiento del impulso formativo. * En alemán en el original. 
aunque más pequeña que la original. ${ }^{27}$ Puesto que "sería un exceso" ${ }^{28}$ suponer que la previsión de la naturaleza ha depositado en ellos disposiciones para desarrollarse en caso de que sean mutilados, la teoría de la evolución debe ser rechazada. Blumenbach considera, consecuentemente, que dicha capacidad de autorreparación que muestran los pólipos debe provenir de una fuerza singular que no poseen los cuerpos inorgánicos, esto es, el impulso formativo que produce las variaciones a partir de los condicionamientos externos de tiempo y lugar.

\subsubsection{Epigénesis o preformismo genérico según Kant}

Pues bien, el modelo de epigénesis desarrollado por el científico natural para explicar el desarrollo de los seres organizados incluye, según hemos vista arriba, dos elementos, es decir, el impulso formativo y los factores físicos externos de tiempo y lugar. Desde el punto de vista de Blumenbach estos elementos son suficientes para explicar la producción de las variaciones de una especie natural. De allí que rechace expresamente la necesidad de suponer las disposiciones y semillas naturales en el desarrollo de lo orgánico.

Ahora, como sabemos, el tipo de epigénesis defendido por Kant conserva el concepto de disposiciones, circunstancia que es base para conjeturar que el filósofo desarrolla un modelo de epigénesis específicamente diferente al de Blumenbach, ${ }^{29}$ esto es, el preformismo genérico. Así, si bien los elementos incluidos por Blumenbach en su teoría de la epigénesis son necesarios, no pueden ser suficientes para completar la comprensión de la reproducción de las variaciones en los seres organizados según lo indica la experiencia pues, según defendería Kant, a fin de dar cuenta de la reproducción de las variaciones de las especies, es decir, la producción de subespecies, es necesario suponer, además, la existencia de ciertas disposiciones.

Ha sido un motivo de discusión entre los críticos la circunstancia de que, a pesar de que la versión de epigénesis que a todas luces parece defender Kant no coincide con aquella de Blumenbach, el filósofo nunca advierta ni sugiera que su concepción de epigénesis tenga alguna diferencia respecto a la desarrollada por el científico de Göttingen. Así, por ejemplo, Richards (2000, p. 12) ha defendido que nos encontramos ante un "malentendido" histórico entre el filósofo y el científico que, no obstante, fue "creativo y productivo". Según señala Bernasconi (2006, p. 74) la "incompetencia científica" de Kant "parece" quedar de manifiesto cuando tanto en la CFJ como en "Sobre el uso" se elogia el concepto de impulso formativo pero se continúa defendiendo un cierto preformismo. Desde nuestro punto de

\footnotetext{
${ }^{27}$ Véase Blumenbach (1791, pp. 87 y ss.). Entre las pruebas del impulso formativo que se mostraría en los animales de sangre caliente, como los hombres, Blumenbach $(1791$, p. 98) menciona el crecimiento de uñas en las extremidades del cuerpo cuando han sido amputadas.

${ }^{28}$ Véase Blumenbach (1791, p. 99).

${ }^{29}$ Alix Cohen $(2009$, p. 22), considera "que la posición de Kant se clasifica mejor como un punto intermedio entre el preformismo y el epigenetismo" pues ella supone "ambos elementos epigenetistas y preformistas”. En este sentido, la posición de Kant es "única".
} 
vista, sin embargo, el silencio de Kant respecto a las diferencias en sus posiciones manifiestas no puede adjudicarse a una carencia de comprensión o de inteligencia por parte de Kant o de Blumenbach. ${ }^{30}$ Si Kant corrigió la teoría de Blumenbach, añadiendo las disposiciones genéricas en la teoría, omitiendo indicar la corrección, se debe, probablemente, a motivos retóricos. La propuesta de introducir las disposiciones genéricas en el interior de la epigénesis ganaría más fácilmente el favor del científico si Kant no se adjudicaba a sí mismo la corrección, sino que la mostraba como una parte integrada o implicada en aquella teoría. De hecho, unos años después, se publicaría un texto titulado Sobre el principio kantiano para la historia natural (1796), cuyo autor, Christoph Girtanner, era un discípulo de Blumenbach, en donde se muestra que los conceptos de semillas y disposiciones de Kant y de impulso formativo de Blumenbach son compatibles, que fue celebrado no solo por Kant, sino también por el científico de Göttingen. ${ }^{31}$ Pero, cabe preguntarnos, ¿por qué Kant corrigió a Blumenbach? ¿Por qué la "epigénesis implica el [cierto] preformismo"? ¿Por qué, "en el origen debe haber alguna dotación (trascendente) y, con ella [...], alguna restricción determinada a la variación de las especies"?32

Que efectivamente es el caso de que Kant integra en un solo modelo los elementos de la epigénesis de Blumenbach y su concepto de disposiciones lo prueba una disquisición dicha al pasar de la sección $\$ 80$ de la CFJ donde Kant boceta el modo como se producirían nuevas configuraciones en el interior de las especies. El filósofo señala:

En lo que se refiere a las modificaciones a las que son sometidos casualmente (zufälligerweise) ciertos individuos (Individuen) de las especies organizadas (organisierte Gattungen), cuando se encuentra que su carácter así modificado (abgeänderter Charakter) es hereditario (erblich) y está incorporado en la fuerza reproductiva (Zeugungskräfte), no puede ser de otra manera juzgado que como el desarrollo oportuno de una disposición conforme a fin originariamente presente en la especie para la autoconservación (Selbsterhaltung). (AA V, p. 420)

\footnotetext{
${ }^{30}$ Como el mismo Bernasconi reconoce (2006, p. 74). Los corchetes son añadido nuestro): "por más atractivo que pueda ser este cuadro [el de la incompetencia científica de Kant], no puede ser sostenido”.

${ }^{31}$ Hemos advertido la relevancia de este texto para comprender la relación Kant/Blumenbach gracias a Zammito (2003, pp. 75 y ss.); Bernasconi (2006, pp. 74-75, 84), sigue también, en este punto, a Jh. Zammito. Como prueba de esto, advirtamos que Girtanner ([1796] 2001, p. 24), señala, por ejemplo, en relación a la historia de las razas de Kant que "una vez que la forma de la estirpe originaria se especifica en una raza, esto es, una vez que el impulso formativo ha recibido una cierta dirección de manera tal que ciertas semillas y disposiciones naturales se desarrollan y otras se contraen [...]". El subrayado es nuestro. De este modo, la forma específica sería una modificación especial del impulso formativo predispuesto en la formación originaria: "el carácter de la raza [esto es, la forma específica], es una modificación especial del impulso formativo”. Los corchetes son añadido nuestro. Girtanner propone así una versión del epigenetismo kantiano compatible con la epigénesis de Blumenbach. Que tanto Kant como Blumenbach habrían acordado con la tesis "compatibilista" de Ch. Girtanner se sigue, como señalan Jh. Zammito y R. Bernasconi en los artículos referidos, de que en la Antropología en sentido prágmático (1798) Kant aprueba expresamente el texto de Girtanner (véase AA VII, p. 320) y así también lo hace Blumenbach en su Manual de historia natural (edición de 1807) y que, previamente, en la edición del mismo texto de 1797, hizo hecho propio el concepto kantiano de raza, que supone el de disposiciones genéricas. Véase Blumenbach ([1779] 1807, p. 25).

${ }^{32}$ Usamos la expresiones de Zammito (2003, p. 88). Los corchetes son añadido nuestro.
} 
Los elementos puestos en juego aquí son (1) las modificaciones casuales sufridas por los individuos de las especies organizadas, (2) las disposiciones de la especie depositadas para la autoconservación de esta e identificadas mediante el criterio de lo hereditario y, finalmente, (3) la fuerza reproductiva (Zeugungskraft). Si advertimos ahora que las "modificaciones casuales" sufridas por los individuos aluden a los factores naturales externos que despiertan las disposiciones según la ocasión, esto es, el contexto geográfico o climático al que están sometidos diferencialmente los miembros de una misma especie, advertimos que los criterios (1) y (3) aquí enumerados coinciden con aquellos que componen el modelo de epigénesis de Blumenbach, esto es, las determinaciones del tiempo y el lugar, por un lado, y el impulso formativo, por otro. La teoría de Kant contiene además, como sabemos, un elemento más (2) correspondiente con las disposiciones. ¿Cómo se integran estos elementos y qué función indispensable cumple cada uno?

Kant acordaría plenamente con Blumenbach en que las condiciones geográficas o climáticas deben jugar un papel en el desarrollo diferencial de los seres orgánicos, esto es, lo que el segundo llamaba la determinación "según el tiempo y [...] las condiciones requeridas del lugar". Las condiciones físicas externas deben dar cuenta, al menos parcialmente, de la dirección hacia la cual se modifican orgánicamente los miembros de una especie. Este es el papel "inconfundible" que se le debe otorgar a la naturaleza en tanto mecanismo en la determinación o condicionamiento que sufren las especies naturales. Los factores físicos externos, sin embargo, no son suficientes para explicar por qué los individuos que han sido alterados por aquellas condiciones pueden reproducir la alteración. Así, Blumenbach debe tener también razón cuando señala que la capacidad de los seres organizados de reproducir las modificaciones debe corresponderse con un impulso formativo singular no reducible a la fuerza mecánica.

Ahora, con todo, estos dos factores no son suficientes para dar cuenta del modo como, según la experiencia, se reproducen las subespecies. Esto es, por qué la fuerza formativa de los seres organizados que han sido alterados por las condiciones externas reproduce necesariamente la variación, por qué una vez ocurrida la diferenciación específica en el interior de una especie no sería posible dar marcha atrás. Como el filósofo sostiene, centralmente, en los textos en los que se ocupa de la cuestión de las razas humanas, ${ }^{33}$ el desarrollo diferencial de la estirpe originaria de nuestra especie en subespecies diferentes es irreversible, esto es, los factores físicos externos no pueden, en la actualidad, hacer mutar los miembros de una raza en otra. Si Blumenbach tuviera razón y los factores físicos externos no tuvieran límites para modificar, una y otra vez, la fuerza formativa, es decir, si ellos pudieran, por sí solos, explicar el origen de las variaciones en tanto subespecies, entonces, los miembros de dichas subespecies que se hubieran trasladado de su hábitat

${ }^{33}$ Véase AA II, p. 441 y AA VIII, p. 98. 
originario deberían poder transformarse, nuevamente, en correspondencia con la nueva geografía. Pero esto es refutado por la experiencia. Desde la perspectiva que defiende Kant, los miembros de dichas subespecies que se han trasladado de paraje no pueden modificar reversiblemente su configuración porque, tan pronto se han despertado las disposiciones genéricas depositadas en la estirpe originaria de la especie, a través del condicionamiento geográfico de origen, la reproducción de las subespecies es necesaria. A partir de entonces, las subespecies dan en herencia, para siempre, los rasgos de su linaje a sus descendientes futuros. Pero esto significa, en consecuencia, que ciertas disposiciones genéricas deben ser supuestas en toda teoría epigenética que pretenda comprender la experiencia. Así, solo el trabajo conjunto de los tres elementos incluidos en la teoría de la epigénesis según Kant puede dilucidar el modo de reproducción de la forma específica de los seres organizados. Por lo tanto, la determinación ocasional del tiempo y el lugar o factores físicos geográficos externos, las disposiciones genéricas de la especie y la fuerza productiva o reproductiva son requeridas a fin de dar cuenta del desarrollo de las variaciones en interior de las especies. Así, Kant concluye proponiendo un modelo propio de comprensión del origen de las variaciones en tanto subespecies en el que convive la epigénesis (de Blumenbach) con cierto preformismo, esto es, el preformismo genérico.

\section{Conclusión}

En lo precedente revisamos la teoría filogenética que se encuentra -en forma de esbozos- en las secciones $\$ \$ 80$ y 81 de la CFJ. Vimos que, en este texto, se encuentra tanto una posición respecto al origen de lo orgánico, en general -en tanto material irreductible al mecanismo de la naturaleza y, por esto, necesariamente referido a una matriz originariamente organizada-, como ciertos argumentos singulares del filósofo respecto al origen de las variaciones en la forma genérica o formación de especies y en la configuración específica o formación de subespecies. A través de una lectura atenta de la letra kantiana y su puesta en discusión con algunos otros textos contemporáneos pudimos poner a la vista una rigurosa y, en parte, original, teoría filogenética kantiana. 


\section{Bibliografía}

Bernasconi, R. (2001), "Who Invented the Concept of Race? Kant's Role in the Enlightenment Construction of Race", en Bernasconi, R.(ed.), Race, Oxford: Blackwell.

Bernasconi, R. (2006), “Kant and Blumenbach's Polyps”, en Eigen, S. y M. Larrimore (eds.), The German Invention of Race, Albany: State University of New York Press, pp. 73-90.

Blumenbach, Jh.F. ([1779] 1807), Handbuch über Naturgeschichte, Göttingen: Johann Chr. Dietrerich.

Blumenbach, Jh.F. (1791), Über den Bildungstrieb und die Zeugunskräfte, Göttingen: Johann Chr. Dietrerich.

Cohen, A. (2006), "Kant on Epigenesis, Monogenesis and Human Nature: the Biological Premise of Anthropology", Studies in History and Philosophy of Science, Part C 37(4): pp. 675-693.

Cohen, A. (2009), Kant and the Human Sciences. Biology, Anthropology and History, Hampshire: Macmillan.

Drews, A. (1894), Kants Naturphilosophie als Grundlage seines Systems, Berlin: Mitscher \& Röstell.

Düsing, K. (1968), Die Teleologie in Kants Weltbegriff, Kantstudien, Bonn: H. Bouvier u. Co. Verlag.

Frigo, G.F. (2009), "Bildungskraft und Bildungstrieb”, en Onnasch, E.-O. (ed.), Kants Philosophie der Natur. Ihre Entwicklung im Opus Postumum und ihre Wirkung, Berlin/New York: Walter de Gruyter, pp. 9-25.

Gerhardt, V. (2002), Immanuel Kant. Vernunft und Leben, Stuttgart: Reclam.

Girtanner, Ch. ([1796] 2001), Über das kantische Prinzip für die Naturgeschichte, Bristol/Virginia: Thoemmes Press.

Herder, Jh.G.v. ([1784-1791] 1924), Ideen für eine Philosophie der Geschichte der Menschheit, Berlín: Deutsche Buch Gemeinschaft. (Versión en español: Herder, J.G., Ideas para una filosofía de la historia de la humanidad, Buenos Aires: Losada, 1959.)

Kant, I. (1902ss), Kants gesammelte Schriften, I-X, hrsg. von der Königlich Preussischen Akademie der Wissenschaften, Berlin, 1902ss. (Ediciones en español consultadas: Kant, I., "Recensión de las Ideas sobre la Filosofía de la Historia de la Humanidad de J.G. Herder", "Sobre el uso de los principios teleológicos en la filosofía" y "Determinación del concepto de una raza humana", en Defensa de la Ilustración, traducción de J. Alcoriza y A. Lastra, Barcelona: Alba editorial, 1999; Kant, I., Crítica de la Facultad de Juzgar, traducción de P. Oyarzún, Caracas: Monte Ávila Editores, 1991.)

Kleingeld, P. (1995), Fortschritt und Vernunft: Zur Geschichtsphilosophie Kants, Würzburg: Königshausen \& Neumann.

Lenoir, T. (1980), "Kant, Blumenbach, and Vital Materialism in German Biology", Isis 71(1): $77-108$.

Lerussi, N. (2011), "Sobre la justificación para introducir el concepto de 'fin natural' (Naturzweck) en la investigación de la naturaleza según la Kritik der Urteilskraft", Kant e-Print. International Journal 6(1): 62-92. ftp://ftp.cle.unicamp.br/pub/kant-e-prints/Vol-6-12011/5-Natalia_Lerussi_fevereiro_2012.pdf

Lovejoy, A. ([1911] 1959), "Kant and Evolution”, en Glass, B. (ed.), Forerunners of Darwin 1745-1859, Baltimore: The John Hopkins Press, 1959, pp. 173-206. 
Löw, R. (1986), Philosophie des Lebendingen. Der Begriff des Organischen bei Kant, sein Gründ und seine Aktualität, Frankfurt: Suhrkamp Verlag.

McFarland, J. D. (1970), Kant's Concept of Teleology, Toronto: University of Edinburgh Press.

McLaughlin, P. (1982), "Blumenbach und der Bildungstrieb. Zum Verhältnis von epigenetischer Embryologie und typologischem Artbegriff", Medizin historisches Journal 17: 357-372.

McLaughlin, P. (1989), Kants Kritik der teleologischen Urteilskraft, Bonn: Bouvier Verlag.

McLaughlin, P. (2010), "Actualism and the Archaeology of Nature”, presentado en el XI Kant Internationaler Kongress, Pisa.

Menzer, P. (1911), Kants Lehre von der Entwicklung in Natur und Geschichte, Berlin: Georg Reimer.

Müller-Sievers, H. (1997), Self-generation: Biology, Philosophy and Literature around 1800, Stanford: Stanford University Press.

Richards, R. (2000), "Kant and Blumenbach on the Bildungstrieb: A Historical Misunderstanding", Studies in the History and Philosophy of Biology and Biomedical Sciences 31: 1132.

Roretz, K. (1922), Zur Analyse von Kants Philosophie des Organisches, Wien: Hölder.

Roth, S. (2008), "Kant und die Biologie seiner Zeit", en Höffe, O. (ed.), Immanuel Kant. Kritik der Urteilskraft, Berlin: Akademie Verlag, pp. 274-287.

Schulze, F. (1875), Kant und Darwin: ein Beitrag zur Geschichte der Entwicklungslehre, Jena: Dufft.

Sloan, Ph. (1976), "The Buffon-Linnaeus Controversy”, Isis 67(3): 356-375.

Stadler, A. (1874), Kants Teleologie und ihrer Erkenntnisstheoretische Bedeutung, Berlin: F. Dümmler.

Tonelli, G. (1954), "La formazioni del testo della Kritik der Urteilskraft", Revue International de Philosophie 8: 423-448.

Ungerer, E. (1922), Die Teleologie Kants und ihre Bedeutung für die Logik der Biologie, Berlin: Verlag von Gebrüder Borntraeger.

Zammito, J. (1992), The Genesis of Kant's Critique of Judgment, Chicago \& London: The University of Chicago Press.

Zammito, J. (1998), “'Method' Versus 'Manner'? Kant's Critique of Herder's Ideen in the Light of the Epoch of Science, 1790-1820", en Adler, H. and W. Koepke (eds.), Herder Jahrbuch/Herder Yearbook, Stuttgart/Weimar: Metzler, pp. 1-25.

Zammito, J. (2002), Kant, Herder and the Birth of Anthropology, Chicago/London: The University of Chicago Press.

Zammito, J. (2003), “'This inscrutable principle of an original organization': Epigenesis and 'Looseness of Fit' in Kant's Philosophy of Science", Studies in History and Philosophy of Science 34A: 73-109.

Zammito, J. (2006), "Policing Polygeneticism in Germany, 1775: (Kames,) Kant, and Blumenbach", en Eigen, S. y M. Larrimore (eds.), The German Invention of Race, Albany: State University of New York Press, pp. 35-54. 